\title{
Chapter 7 \\ Towards Understanding Acquisition Conditions Influencing Finger Vein Recognition
}

\author{
Simon Kirchgasser, Christof Kauba and Andreas Uhl
}

\begin{abstract}
Finger vein biometrics are of growing influence in daily life high-security applications like financial transactions. Several application areas of finger vein recognition systems exhibit different environmental and non-environmental conditions, e.g. changes in temperature, illumination, humidity and misplacement of the finger. Experience in the application of various biometrics (e.g. fingerprints, iris, face) shows that acquisition condition changes may affect the recognition process. No systematic analysis on the impact of those condition changes influencing the performance of finger vein recognition systems has been conducted so far. In this chapter, 17 possible acquisition conditions are identified, described and a first proper investigation regarding their influence on the recognition process is performed. This investigation is done based on several well-established finger vein recognition schemes and a score distribution analysis. The insights gained in this chapter might help to improve finger vein recognition systems in the future. The first results reveal several acquisition conditions that significantly decrease the recognition performance. Especially external illumination condition changes and finger tissue temperature variation shows a severe impact. On the other hand, slight misplacement of the finger and sport activities (in particular cycling) has hardly any influence on the recognition performance.
\end{abstract}

Keywords Finger vein recognition - Biometric data acquisition conditions • Biometric performance evaluation $\cdot$ External influences $\cdot$ Subject-related influences

\footnotetext{
S. Kirchgasser $(\bowtie) \cdot$ C. Kauba $\cdot$ A. Uhl

Department of Computer Sciences, University of Salzburg, Jakob-Haringer-Str. 2, 5020 Salzburg, Austria

e-mail: skirch@cs.sbg.ac.at

C. Kauba

e-mail: ckauba@cs.sbg.ac.at
}

A. Uhl

e-mail: uhl@cs.sbg.ac.at

(C) The Author(s) 2020

A. Uhl et al. (eds.), Handbook of Vascular Biometrics, Advances in Computer Vision and Pattern Recognition, https://doi.org/10.1007/978-3-030-27731-4_7 


\subsection{Introduction}

In various biometric applications, e.g. iris or fingerprint recognition, studies have been performed to describe and quantify varying acquisition conditions. Concerning challenging aspects in iris biometrics, an overview is given in [6]. In fingerprint applications, various studies exist too. Some important ones related to the current study can be found in [23, 25, 27]. In [25], the authors, Simon-Zorita et al., evaluated an automatic minutiae-based fingerprint verification system on the MCYT Fingerprint Database [19], which includes several variabilities of factors that occur in a typical daily acquisition process. The effects of a controlled image acquisition (done under ideal environmental, subject and internal conditions) on fingerprint matching results have been investigated in [23] using various methods to enhance the quality of imprints acquired under challenging conditions (including changes in ambient temperature and humidity). Finally, in [27], Stewart et al. took rugged environmental conditions, especially cold weather, into account during tests regarding the performance of fingerprint recognition technology. The experimental results show no critical deficiencies in the recognition performance regarding the considered challenging environmental conditions. Unfortunately, the authors only reported the results for the false rejection rate (FRR), which indicated no relationship between the recognition results and the cold weather condition.

According to the investigations done in other biometric fields, it is necessary to identify, describe and quantify environmental- and non-environmental-based conditions which could influence finger vein (FV) recognition systems. This consideration might have some impact on the performance evaluation, improvement and more frequent employment of FV recognition systems in daily life. Prior to this, several conditions have to be selected and suitable data needs to be acquired. Some considered environmental condition changes will include variations in the skin humidity or in the finger temperature, placing a light source in front of the scanner or putting the finger into a water bath to soften the skin. Subject-related condition changes might exhibit finger misplacement (e.g. finger is not covering the scanner light source entirely), usage of hand or sun lotion and sport activities. A detailed description of all investigated variations is given in Sect. 7.4.

The rest of this chapter is organised as follows: In Sect.7.2, a detailed review on related work and research results is given. Then the scanner devices used during the data acquisition and the considered conditions are described in Sect.7.3 and 7.4, respectively. Section 7.5 illustrates the experimental setup. The performance evaluation together with a discussion of the results are presented in Sect. 7.6. Finally, Sect. 7.7 concludes this chapter along with an outlook on future work. 


\subsection{Varying Acquisition Conditions-A Challenging Aspect in Research and Practical Applications}

Varying environmental- or subject-related variations in acquisition conditions should not influence the security and reliability of biometric authentication solutions. This is vital for FV recognition systems as well because they are used in high-security applications, such as financial services, automated teller machines (ATMs) and for securing online banking transactions. Especially FV scanners deployed in ATMs can be influenced by varying environmental conditions easily as those machines are often located outside and thus prone to changes in illumination, temperature and humidity (note: in Japan they are usually inside the bank building). A user might be enrolled inside the bank building but the ATM at which the authentication is performed might be located outside the building and not inside. Thus, there might be direct sunlight shining on the FV scanner device situated at the ATM where the user wants to withdraw money after the authentication. Furthermore, it is possible that the user presents his/her finger in a slightly different way as he/she did during the enrolment. These varying acquisition conditions can cause severe problems in real-life applications because the accuracy and reliability of biometric recognition systems, in particular, of FV-based systems, are undermined.

As motivated in the introduction, several factors can affect the recognition accuracy of vascular pattern based authentication systems. First of all, inherent biological factors may influence the FV recognition process. According to Kumar et al. [12], the quality of finger vein images can vary across the population for different users. This statement was postulated only and was not proven by empirical experiments. These quality variations might be caused by factors like gender (e.g women usually have thinner fingers than men), daily physiological composition [28], medical conditions (e.g. thickness of persons' tissue layers may change due to fat [28]), anaemia, hypotension, hypothermia and various other aspects as discussed in [5, 24].

Another major impact is related to the optical component used in the applied scanner devices. The NIR light, used to render the vein structure visible in the captured images, is absorbed, reflected and scattered during the penetration of the humans' tissue. Light scattering imposes the most severe impact of these three aspects. In biomedical imaging research, these factors have been extensively investigated. Dhawan et al. [9] reviewed several models enabling the propagation of visible and NIR light photons in biological tissue for biomedical and clinical application. This study included (among others) the usage of transillumination NIR light in clinical practice. The results of using NIR transillumination have shown a significant potential in diagnostic applications but there are still difficulties due to scattering of the NIR light in the biological tissue. A more specific application area was discussed by Bashkatov et al. [4]. The authors focused on the description of optical properties of human skin, subcutaneous and mucous tissues using light exhibiting different wavelengths. They reported specific light scattering and absorption coefficients for each of the considered tissues. These coefficients vary highly among the investigated modalities. Consequently, it is not possible to neglect the aspect of biological influ- 
ences in FV applications but they are usually considered as a given constant factor, which has to be covered by various image preprocessing, restoration and enhancement techniques [29]. Further results regarding optical influences are reported by Baranoski et al. [3]. They simulated light interaction effects within the human skin by the application of computer graphics techniques. The authors only investigated effects introduced by the first layer of the finger tissue. Furthermore, a discussion regarding light scattering influences on the FV recognition performance was not given. Another study by Cheong et al. [7], neglecting real-life recognition aspects, used mathematical models with respect to optical coefficients describing various human tissue layers. The authors described and discussed optical properties of blood vessels (in particular the aorta), liver and muscle tissue. There are several other nonbiological factors which might have an impact on the recognition performance of FV-based systems as well. These non-biological factors can be grouped into internal factors and external factors.

At first, we focus on various internal factors. The most important ones are those which are introduced by the biometric scanner device itself. The equipped illumination source might be too bright or too dark either due to a wrong specification of the illumination source, due to problems with the brightness control module or due to fingers being too thick or too thin. Furthermore, the equipped camera module might be sensitive to ambient light changes as sunlight contains NIR light. Another influencing factor is a high sensitivity to dust which affects the camera as well. Both of these image sensor specific internal factors lead to a decrease in the FV image quality. Fortunately, it is possible to cope with these problems by changing the scanner setup or adding additional components. To reduce the sensitivity to ambient light, a daylight blocking filter or a housing around the scanner can be mounted additionally. Moreover, it is possible to use thermal FV imaging as well. Thermal vein pattern images are insensitive to ambient light changes under a wide range of lighting conditions as reported in [15]. However, this adds the necessity to cope with other difficulties like problems with varying ambient temperature or changes in the human body temperature as discussed by the authors of [13].

A recent study of Kauba et al. [11] investigated the impact of several internal factors. The authors considered sensor noise, sensor ageing related defects as well as other image distortions which are potentially present in real-life hand- and FVpattern acquisition conditions. Moreover, they considered different levels of artificial distortions. Such artificial distortions might be present in practical hand vein applications but fortunately the authors reported that the evaluated hand vein recognition schemes show a high robustness against the investigated influences. In our present study, we ensure that the scanner setup is optimal in order to reduce the number of distorting aspects due to internal factors to a minimum.

The class of external factors can be separated into two independent categories: environmental aspects and subject-related (non-environmental) aspects. These two classes of external influencing factors are the main scope of the current work. Nonenvironmental-related factors include finger movement during the image acquisition and misplacement of the finger on the scanner device, including tilt, bending and rotation of the finger along its longitudinal axis. Matsuda et al. [17] and Yang et 
al. [30] investigated the aforementioned finger tilt and non-planar finger rotation. In both works, the authors introduced recognition schemes which are able to deal with non-planar finger rotation up to a certain degree. Matsuda et al. stated that their deformation-tolerant feature point matching scheme is able to cope with nonplanar finger rotation up to $\pm 30^{\circ}$. Yang et al. did experiments with their proposed recognition scheme regarding finger rotation as well but they did not state to what extent of rotation their scheme is robust against. Furthermore, both authors claim that their proposed schemes show a high level of robustness against elastic deformations of the acquired vein patterns. In a more recent study by Prommegger et al. [20], the effects of longitudinal rotation within FV datasets have been investigated. The authors prove experimentally that longitudinal finger rotation poses a severe problem in FV recognition as the resulting vein image may represent entirely different patterns due to the perspective projection. This variation in the perspective projection results in a severe performance decrease using simple recognition schemes if more than $\pm 10^{\circ}$ rotation is contained in the images. More sophisticated FV recognition schemes are able to handle up to $\pm 30^{\circ}$ without leading to a performance decrease.

Apart from finger movement related investigations, there are several studies showing that various environmental factors have a crucial impact on FV recognition. The most important aspects are varying ambient light conditions [26], light scattering effects [29] as well as ambient temperature changes as discussed in [18]. Miura et al. [18], Song et al. [26] and Yang et al. [29] discuss these distortions only briefly without any further performance experiments targetting the influence of these variations. However, in [18], an analysis was done in order to quantify the influence of ambient temperature changes while a more robust matching scheme was introduced. The authors proposed a scheme that calculates local maximum curvatures in cross-sectional profiles of an FV image. Thus, fluctuations regarding vein width and brightness, introduced by, e.g. ambient temperature changes and physical activities involving the fingers/hands, are reduced during the feature extraction. The following studies introduce different aspects of complicating factors in FV recognition: In [14], bifurcations and ridge endings (originally fingerprint minutiae types) are selected for image alignment followed by a unique FV code extraction, which is based on local binary patterns. The minutia points' extraction can easily be influenced by distortions introduced during the acquisition of the FV pattern. However, the authors only mention that the number and the positions of minutia points may vary among the acquired data due to possible changes in finger location, different posture and varying lighting conditions. Hashimoto [10] mentions that variations within the FV data need to be controlled. Body metabolism changes, brightness fluctuations due to individual variations in finger size or lighting conditions are discussed as major influencing factors without considering these aspects in the performance evaluation of the recognition process. A recent study by Qin et al. [21] states that a proposed convolutional neural network $(\mathrm{CNN})$ on the one hand makes the recovery of missing vein patterns possible and on the other hand is able to suppress noise resulting from segmentation in case a low-quality image is processed. The detection of such low-quality images is mandatory in the first place and can be done by the application of several available FV quality measures [22, 28]. 
The main aspects of these studies have in common are (a) challenging aspects are only seen as a given problem and therefore no further investigation is dedicated to them and (b) there are only very few studies available which try to describe the influence on the recognition performance. One recent investigation focusing on the impact of different meteorological aspects like temperature, humidity, atmospheric pressure and wind was performed by Zheng et al. [33]. Based on the experimental results the authors concluded that ambient temperature is the most significant factor. They further proposed two methods, dynamic template selection and a so-called threshold adjustment, to reduce the impact of ambient temperature changes during the recognition process.

For the further development of FV recognition systems, it would be of great value to understand which acquisition variations are causing which amount of degradation in the FV recognition process. This work is dedicated to this important aspect.

\subsection{Deployed Scanner Devices}

It is possible to categorise the various types of FV scanners into several classes: contactless/full contact scanners and LED/laser light devices are the most important ones. All available COTS FV scanners are full contact and based on transillumination using LEDs. According to the fact that almost all COTS FV scanners do not enable direct access to the raw vein images they acquire, they would be only of little use during this study as we need to conduct a recognition toolchain on the raw vein images to evaluate the used algorithm performance on the dataset containing influenced FV images. This evaluation is mandatory to improve the algorithm's robustness against varying acquisition conditions and could not be done by using COTS FV scanners.

Two different types of illumination can be distinguished. Their classification is based on the relative positioning of the camera module, the finger and the equipped light source. The first method is called light transmission or transillumination and the second one is called reflected light. NIR LEDs as well as NIR laser modules can be deployed for both illumination setups.

In the transillumination concept, the light source and the image sensor are placed on opposite sides of the finger that is acquired. The emitted NIR light passes through the finger, where it is absorbed by the blood vessels and is captured by the imaging module on the opposite side. The veins, or to be more precise the blood vessels, appear as dark lines in the images. We decided to deploy only the transillumination concept as the FV image quality is better compared to the quality of images acquired using reflected light.

Two self-designed FV scanner devices were used to acquire the FV images and are similar to the scanners discussed in Chap.3. The main difference is that the deployed devices are not able to capture FV images of three fingers at the same time. Only one finger after the other can be processed to acquire the blood vessel pattern. However, one scanner is equipped with a NIR laser illumination module and one with an LED illumination module, both are arranged in a strip placed underneath 

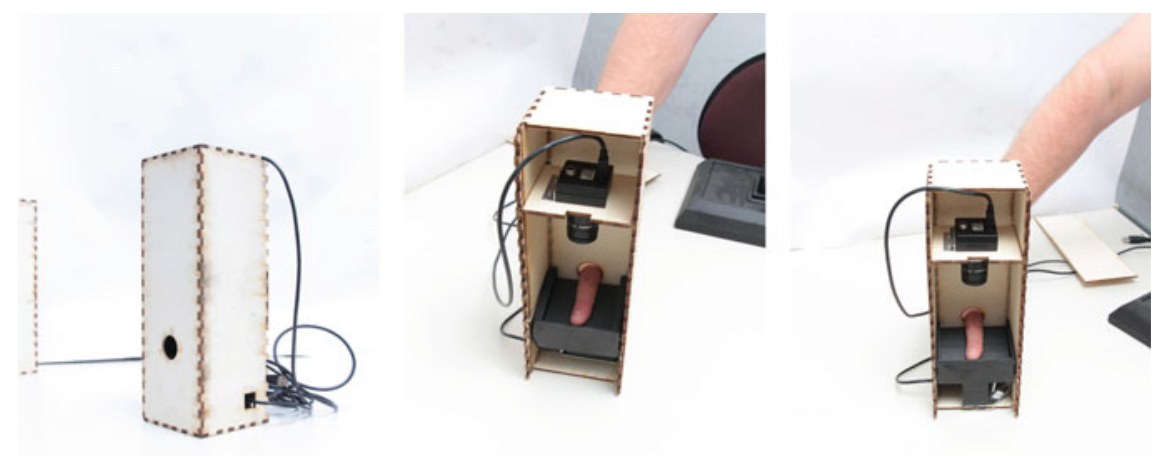

Fig. 7.1 NIR LED and laser-based finger vein scanner (camera on top and finger at bottom)

the finger support. Both scanners are designed in the same way and are based on the transillumination principle. The NIR LEDs have a peak wavelength of $860 \mathrm{~nm}$, while the laser modules have a peak wavelength of $808 \mathrm{~nm}$. The captured FV images have a resolution of $1280 \times 1024$ pixels and are stored as 8-bit greyscale images. Furthermore, an additional NIR pass-through filter is mounted to reduce the influence of ambient light and to improve the quality of the acquired images. The used image sensor is an industrial NIR-enhanced camera, IDS Imaging UI-1240ML-NIR [2], equipped with a Fujifilm HF9HA-1B $9 \mathrm{~mm}$ wide-angle lens [1]. The scanners are depicted in Fig. 7.1.

A wooden box (left image) surrounds all parts of the device, including the camera module, the NIR light strip and the finger support, in order to reduce the amount of ambient light to a minimum. The middle and right images of Fig. 7.1 reveal the light transmission concept. The camera is placed on top, the finger can be seen in the middle placed on the finger support, and the illumination module is located at the bottom of the wooden box. In the middle image, the LED-based version of the scanner is shown, while the right image represents the laser-based one. Compared to other FV scanners this positioning concept is different and results in the visualisation of the blood vessel patterns which are located at the upper side of the finger.

\subsection{Finger Vein Acquisition Conditions Dataset}

Currently, there is no publicly available dataset FV that exhibits various environmental and non-environmental acquisition conditions. Thus, we established our own subset, including different environmental and subject-related acquisition variations.

In general, every biometric authentication system consists of an enrolment and a verification stage. During the mandatory enrolment stage, the considered biomet- 
ric pattern of the user is acquired under controlled and supervised conditions. This ideal situation will likely not be present during the second stage of the authentication process, the verification. Each time the user wants to authenticate him-/herself, he/she has to present his/her biometric trait which is then acquired once again. This biometric data acquisition during authentication might be performed under different environmental conditions.

To simulate a more realistic enrolment and authentication procedure, the data acquisition was performed under a controlled environment. At first, the enrolment was done under optimal and stable environmental conditions and a correct subject's scanner handling was ensured. The authentication was simulated in a second acquisition session, on the same day by manually introducing one specific condition change (environmental or subject related) at a time. This controlled acquisition environment ensured the capturing of data acquired under exactly one varying aspect (disregarding other, additional influences).

The first subset included in our database "reflects" this enrolment subset. This first subset is exhibiting no distorting aspect which is important as a reference and for the baseline recognition evaluation.

Besides the enrolment subset we acquired a total of 17 subsets exhibiting acquisition variations. Seven of these subsets have been acquired under varying environmental conditions and 10 subsets exhibit subject-related condition changes. We aimed to include the most promising aspects relating to acquisition conditions present in real-life deployments of FV recognition systems.

The entire data acquisition was done indoors with indirect illumination (fluorescent lamps at the ceiling) only. The humidity and temperature in the room have been kept stable during the entire process at a level of $23{ }^{\circ} \mathrm{C}$ and approximately $75 \%$ humidity. Each of the investigated acquisition conditions are introduced intentionally and manually during the acquisition of the specific subset independent from the others. If the humidity of the fingers was changed or sports activity was performed before the acquisition process, the necessary alternations were introduced in a different room or outside (sports) to preserve stable acquisition conditions in the room, where the FV device was placed. This reduces the influence of other conditions than the desired acquisition condition changes. In the following, each of the considered acquisition conditions is described in detail and example images are displayed in Figs. 7.2 and 7.3 for the laser scanner and the LED scanner, respectively. The top-left image in both figures corresponds to the enrolment stage (subset base). The other images, all captured from the same finger, are exhibiting one acquisition condition variation each:

base: $\quad$ baseline (no distortion included)

Environmental Condition Changes :

humid: increasing the humidity by putting the fingers next to a humidifier placed in a neighbouring room to prevent humidity changes in the acquisition room. 

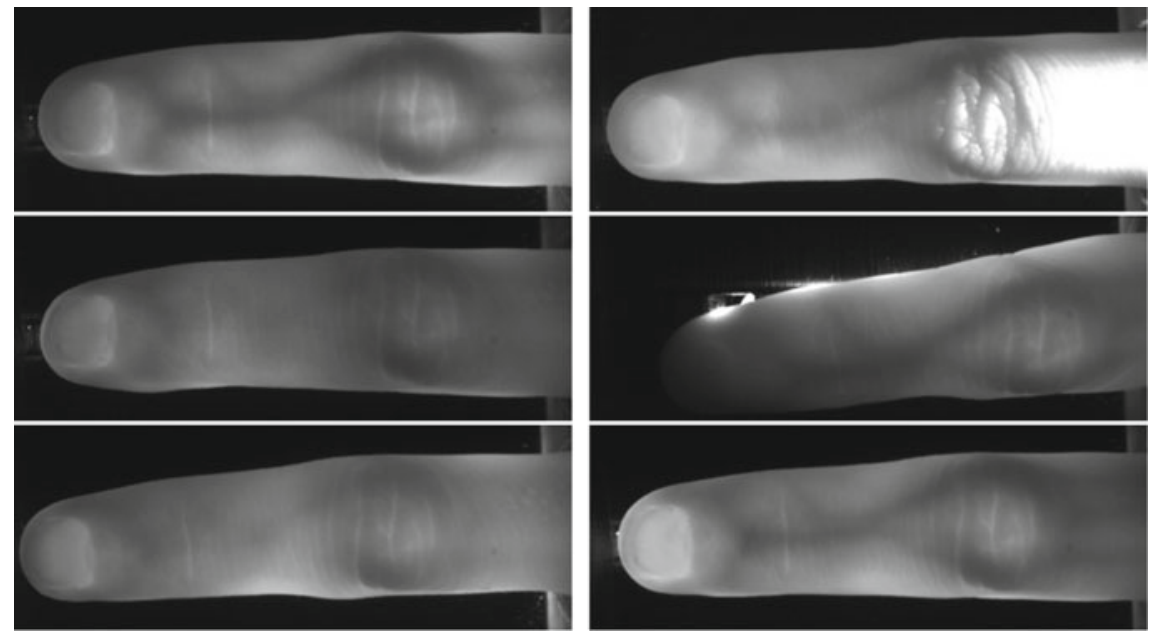

Fig. 7.2 Laser FV scanner images (same finger) of all subsets-1. row: base (left), light (right)-2. row: temp -5 (left), badpl (right)-3. row: sunlot (left), cycle (right)
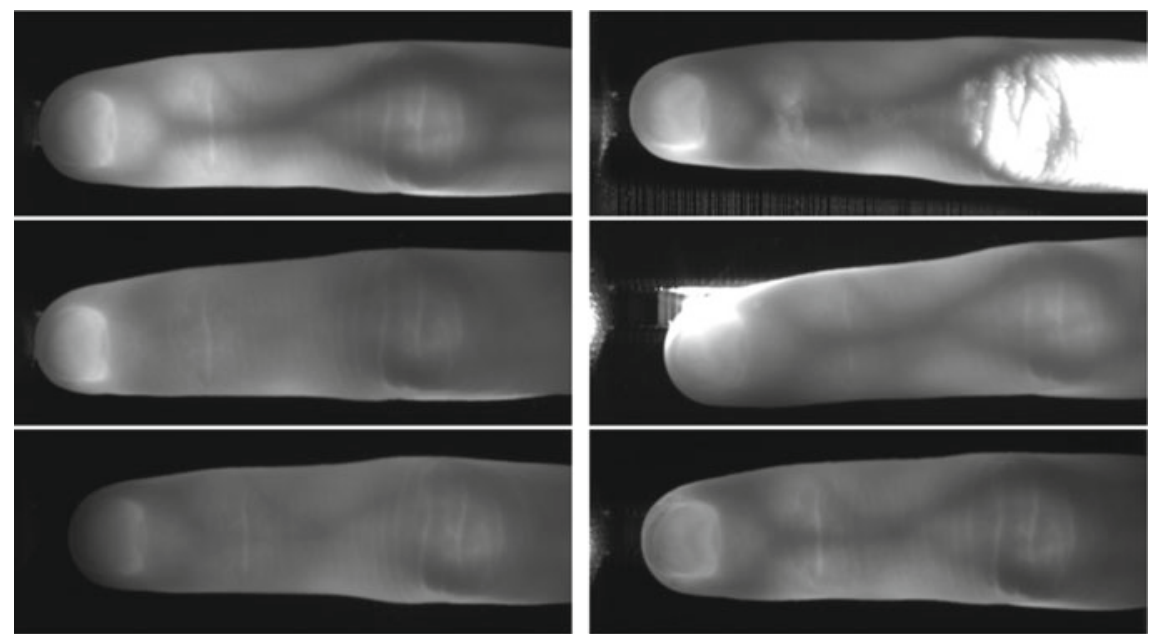

Fig. 7.3 LED FV scanner images (same finger) of all subsets-1. row: base (left), light (right)-2. row: temp -5 (left), badpl (right)-3. row: sunlot (left), cycle (right)

light: $\quad$ placing a battery torch with a low light emission intensity in front of the scanner

dark: $\quad$ shutting off the room light.

temp - 5: lowering the finger's temperature, introduced by an ice water bath where the finger was put in for $5 \mathrm{~min}$. 
temp $+5:$ increasing the finger's temperature introduced by a hot water bath where the finger was put in for $5 \mathrm{~min}$.

skin 10: putting the fingers in a body temperature water bath for $10 \mathrm{~min}$ (this does not alter the temperature of the finger, but the skin structure is influenced-besides, the fingers are dried afterwards to avoid influences by the wet fingers).

skin25: putting the finger in a body temperature water bath for $25 \mathrm{~min}$.

\section{Subject Related Condition Changes :}

up5: $\quad$ putting both arms straight upside to the vertical position and hold this position for $5 \mathrm{~min}$.

up 10: $\quad$ putting both arms straight upside to the vertical position and hold this position for $10 \mathrm{~min}$.

tremb: $\quad$ imitating that the finger placed inside the scanner is trembling.

badpl: $\quad$ placing the finger inside the scanner in a way that the light source is not covered entirely.

bend: $\quad$ bending the finger inside the scanner.

tip: $\quad$ tilting the finger forward, so only the fingertip is placed on the light strip (the tilting angle is about 10 degrees, and hence most of the illumination goes through the finger's tissue).

trunk: tilting the finger backwards, so the fingertip is not touching the light strip but the trunk touches the scanner surface (the tilting angle is about 10 degrees, and hence most of the illumination goes through the finger's tissue).

handlot: applying hand lotion to the finger's surface.

sunlot: applying sun lotion to the finger's surface.

cycle: cycling for about $20 \mathrm{~min}$ before the image acquisition.

The subsets represented by the given example images have been selected because they are likely to exhibit severe impact in practical applications. From a visual point of view, it can be concluded that variations as displayed by the middle and last image located in the left column and the first two images in the right column could cause some problems during the recognition process. The visibility of the blood vessel patterns in each of these four images is clearly suppressed by the variation as compared to the top-left baseline image. During the experimental discussion, we will come back to these subsets.

There is currently no detailed knowledge of which variation introduces the most severe impact in terms of recognition accuracy degradation. This study is the first of its kind focusing on environmental- and non-environmental (subject)-related acquisition conditions in the scope of FV recognition. It can serve as a basis for further investigations on this topic. Only two subjects have been acquired due to the very time-consuming process of acquiring the images. This leads to a total of 60 images per condition ( six fingers per subject-index, middle and ring finger, each finger was 
acquired five times). Although the number of images per finger is limited, there is still sufficient data available to quantify the impact of the various distortions.

\subsection{Finger Vein Recognition Toolchain and Evaluation Protocol}

The first part of the FV recognition toolchain is the FV preprocessing to enhance the vein pattern quality. This process consists of Region-of-Interest (ROI) extraction, image filtering and enhancement. At first, the ROI is extracted from the input images using edge detection. Afterwards, the vein pattern's visibility is enhanced by the application of various techniques: High Frequency Emphasis Filtering (HFE) [32], Circular Gabor Filter (CGF) [31] and CLAHE (local histogram equalisation) [34]. The second part of the FV recognition toolchain includes feature extraction and feature comparison resulting in a list of comparison scores. We selected two well-established binarisation-type feature-extraction methods, Maximum Curvature (MC) [18] and Principal Curvature (PC) [8], as well as one key point (SIFT) [16] based method with additional key point filtering for the feature extraction. The binarisation-type methods aim to extract the vein pattern from the background resulting in a binary output image, which represents the extracted features. After the feature extraction is completed, it is followed by a comparison of these binary images as proposed by [18].

To obtain the comparison scores, all possible genuine and impostor comparisons are performed. This is done by comparing each image against all remaining ones which finally results in 120 genuine and 1650 impostor comparisons per subset. The comparison scores can be downloaded from: http://www.wavelab.at/sources/ Kirchgasser19a/. We utilised the PLUS-OpenVein SDK which was presented in Chap. 4 of this book. A publicly available implementation of the complete processing toolchain can be downloaded from: http://www.wavelab.at/sources/OpenVeinToolkit/.

We have selected several well-established measures to quantify and categorise the different acquisition conditions. Thus, the average genuine score values (avGen) as well as the average impostor score values (avImp) have been calculated first. The tendency of the avGen and avImp values gives a first hint which acquisition condition might have the most severe impact on the recognition process. The avGen and avImp are listed in the result Tables 7.1 till 7.5 in the second and third columns. Additionally, we have selected the equal error rate (EER), the lowest FNMR for FMR less or equal to $1 \%\left(\mathrm{FMR}_{100}\right)$, the lowest FNMR for FMR less or equal to $1 \%\left(\mathrm{FMR}_{1000}\right)$, Zero Match Rate (zFMR) and Zero Non-Match Rate (zFNMR) as performance measures. These values will be listed in the result tables in columns four till eight given in percentage. 

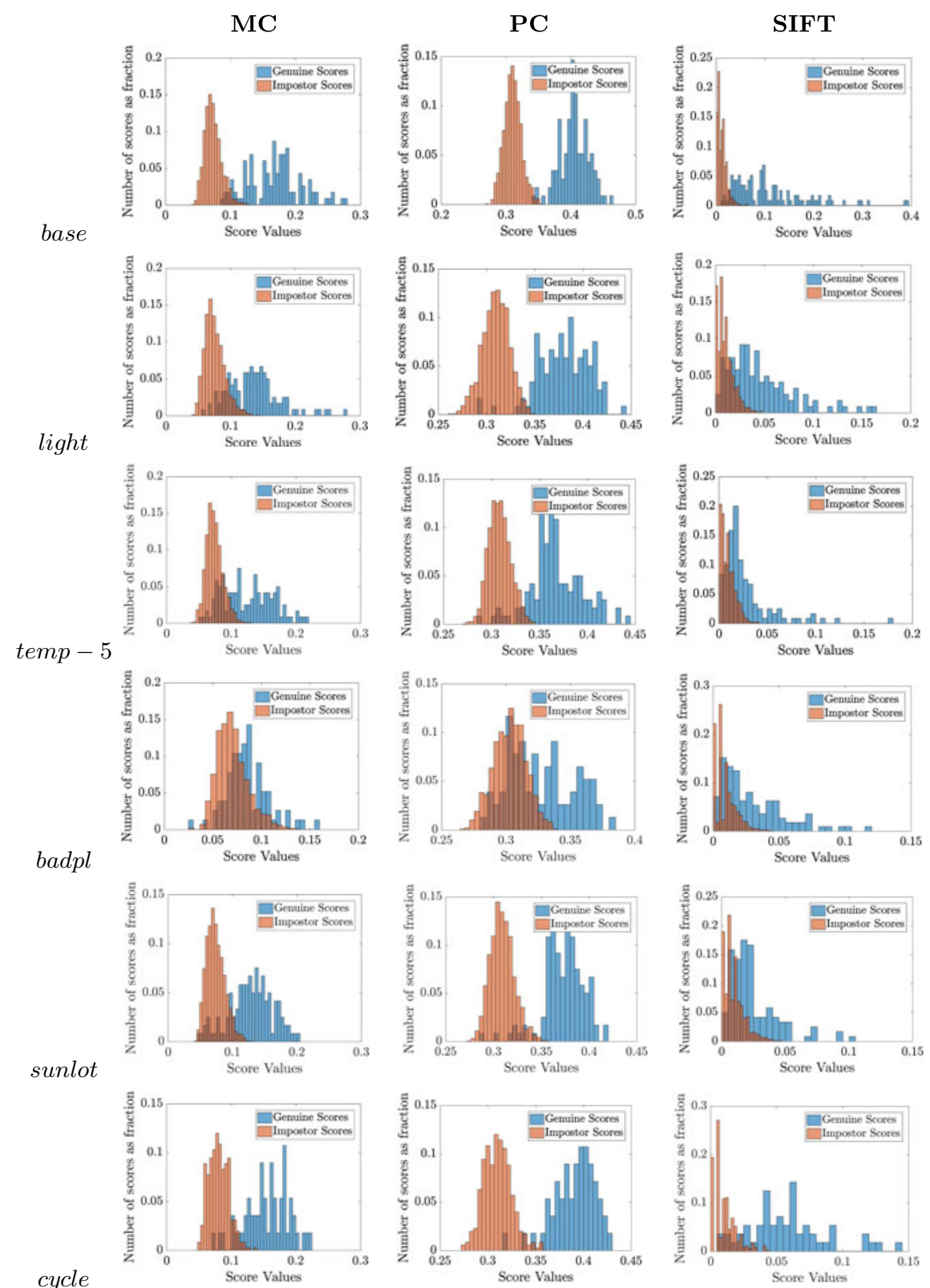

Fig. 7.4 Laser subsets matching score distributions MC (left column), PC (middle column) and SIFT (right column): 1. row: base-2. row: light-3. row: temp $-5-4$. row: badpl-5. row: sunlot and 6. row: cycle 

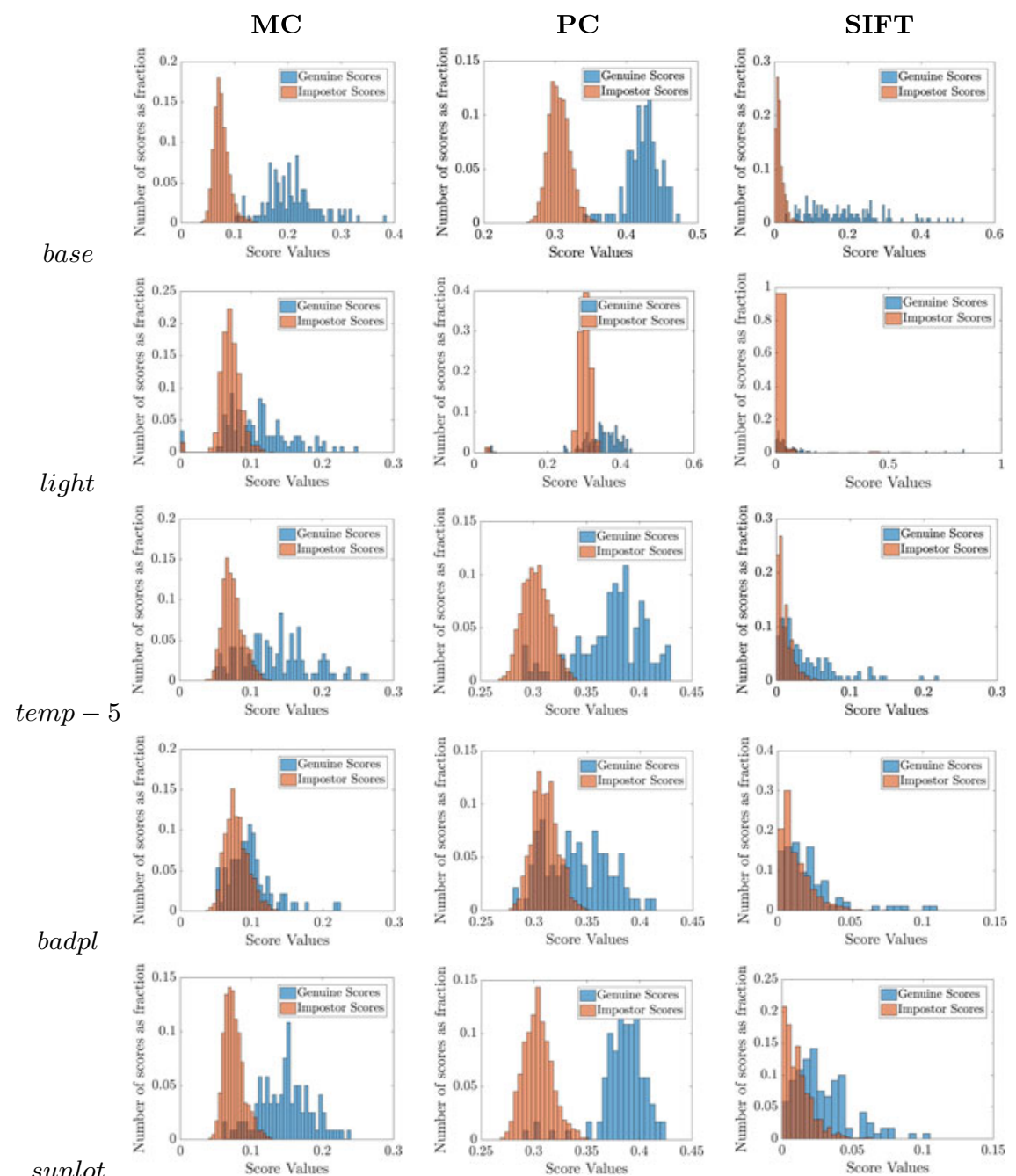

sunlot
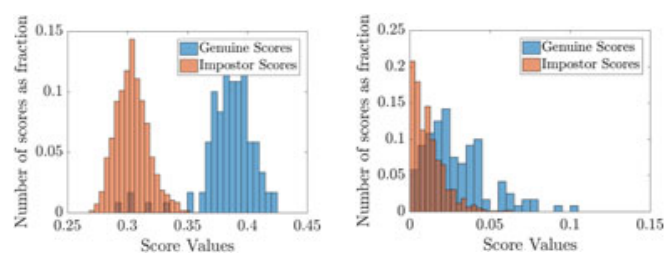

cycle
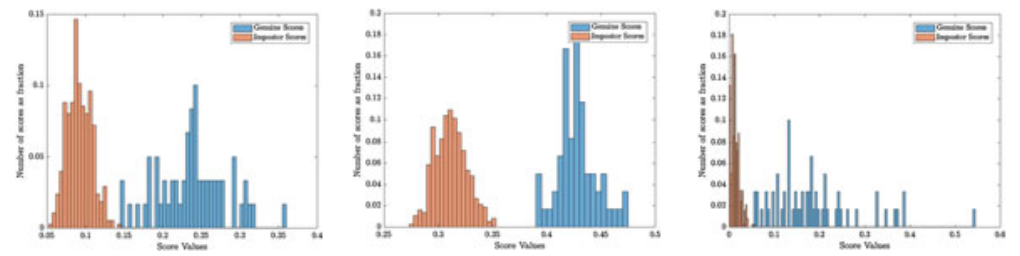

Fig. 7.5 LED subsets matching score distributions MC (left column), PC (middle column) and SIFT (right column): 1. row: base-2. row: light-3. row: temp - 5-4. row: badpl-5. row: sunlot and 6. row: cycle 
Table 7.1 Performance evaluation results for MC using the laser scanner data

\begin{tabular}{|c|c|c|c|c|c|c|c|}
\hline Name & avGen & avImp & EER & $\mathrm{FMR}_{100}$ & $\mathrm{FMR}_{1000}$ & zFMR & zFNMR \\
\hline \multicolumn{8}{|l|}{ Baseline } \\
\hline base & 0.17 & 0.07 & 4.15 & 10.3 & 16.4 & 18.9 & 12.41 \\
\hline \multicolumn{8}{|c|}{ External condition changes } \\
\hline humid & 0.14 & 0.07 & 10.82 & 25.8 & 32.5 & 48.3 & 88.7 \\
\hline light & 0.12 & 0.07 & 12.48 & 33.3 & 50.8 & 61.66 & 85.7 \\
\hline dark & 0.13 & 0.07 & 13.29 & 33.0 & 47.5 & 47.5 & 97.2 \\
\hline temp -5 & 0.12 & 0.07 & 18.34 & 39.2 & 51.7 & 54.2 & 96.9 \\
\hline temp +5 & 0.13 & 0.07 & 11.45 & 30.8 & 43.3 & 44.2 & 73.7 \\
\hline $\operatorname{skin} 10$ & 0.12 & 0.07 & 11.66 & 34.2 & 53.3 & 55.8 & 92.3 \\
\hline $\operatorname{skin} 25$ & 0.13 & 0.07 & 8.39 & 25.8 & 43.3 & 43.3 & 93.3 \\
\hline \multicolumn{8}{|c|}{ Subject-related condition changes } \\
\hline up5 & 0.13 & 0.07 & 45.64 & 7.5 & 16.6 & 19.3 & 99.8 \\
\hline up 10 & 0.12 & 0.07 & 43.44 & 6.9 & 15.5 & 18.1 & 98.3 \\
\hline tremb & 0.11 & 0.07 & 18.65 & 54.4 & 79.4 & 86.6 & 93.0 \\
\hline badpl & 0.09 & 0.07 & 22.51 & 93.5 & 97.4 & 97.4 & 99.9 \\
\hline bend & 0.1 & 0.07 & 12.14 & 20.6 & 33.6 & 45.6 & 97.2 \\
\hline tip & 0.13 & 0.07 & 9.40 & 61.3 & 88.0 & 96.5 & 62.0 \\
\hline trunk & 0.1 & 0.07 & 20.85 & 82.5 & 98.3 & 98.3 & 89.7 \\
\hline handlot & 0.14 & 0.07 & 15.81 & 30.8 & 40.8 & 43.3 & 86.4 \\
\hline IDsunlot & 0.12 & 0.07 & 11.66 & 28.3 & 36.6 & 45.8 & 98.2 \\
\hline cycle & 0.16 & 0.07 & 8.60 & 12.5 & 30.3 & 30.3 & 67.4 \\
\hline
\end{tabular}

\subsection{Experimental Results Analysis}

In the following, all results are discussed in detail together with the general trend of the different acquisition conditions highlighted by the worst and best results which have been achieved.

Figures 7.4 and 7.5 display the score distribution plots for the laser-based and the LED-based scanner, respectively. Each column of both figures corresponds to a single recognition scheme: the left column shows the values for $\mathrm{MC}$, the middle column displays the results obtained using PC and the right column depicts the SIFT results. Each row is dedicated to one subset that has been described before. These subsets have been selected because they are likely to exhibit severe impact in practical applications.

The calculation of the baseline results is a special case: they are calculated by comparing subset base with itself, while for other results the comparison is always done between subset base as gallery and one of the probe subsets humid till cycle. This setup is in regards with the usual enrolment/authentication scenario in real-life applications. However, it must be mentioned that the number of performed comparisons is lower for the baseline experiments. As comparisons with the same images 
Table 7.2 Performance evaluation results for PC using the laser scanner data

\begin{tabular}{|c|c|c|c|c|c|c|c|}
\hline Name & avGen & avImp & EER & $\mathrm{FMR}_{100}$ & $\mathrm{FMR}_{1000}$ & zFMR & zFNMR \\
\hline \multicolumn{8}{|l|}{ Baseline } \\
\hline base & 0.4 & 0.31 & 0.86 & 0.9 & 2.6 & 2.6 & 1.2 \\
\hline \multicolumn{8}{|c|}{ External condition changes } \\
\hline humid & 0.38 & 0.31 & 3.52 & 4.2 & 5.0 & 5.8 & 61.1 \\
\hline light & 0.38 & 0.31 & 3.33 & 5.0 & 5.8 & 9.2 & 91.8 \\
\hline dark & 0.37 & 0.31 & 0.01 & 10.8 & 11.7 & 11.7 & 91.8 \\
\hline temp -5 & 0.37 & 0.3 & 6.67 & 8.3 & 14.2 & 15.8 & 96.8 \\
\hline temp +5 & 0.38 & 0.31 & 2.49 & 2.5 & 3.3 & 5.8 & 92.9 \\
\hline $\operatorname{skin} 10$ & 0.37 & 0.31 & 4.99 & 9.2 & 10.8 & 15.8 & 68.3 \\
\hline $\operatorname{skin} 25$ & 0.37 & 0.31 & 2.49 & 2.5 & 5.8 & 5.8 & 73.8 \\
\hline \multicolumn{8}{|c|}{ Subject-related condition changes } \\
\hline up5 & 0.38 & 0.31 & 40.01 & 7.1 & 15.9 & 18.9 & 97.3 \\
\hline up 10 & 0.36 & 0.31 & 40.56 & 6.7 & 15.1 & 17.3 & 96.6 \\
\hline tremb & 0.37 & 0.31 & 12.51 & 13.4 & 21.4 & 24.1 & 95.1 \\
\hline badpl & 0.34 & 0.3 & 19.85 & 57.1 & 70.1 & 70.1 & 95.2 \\
\hline bend & 0.34 & 0.31 & 12.92 & 17.2 & 18.9 & 21.5 & 99.7 \\
\hline tip & 0.38 & 0.31 & 5.97 & 67.6 & 77.6 & 77.9 & 87.6 \\
\hline trunk & 0.35 & 0.31 & 16.55 & 55.0 & 70.8 & 88.3 & 99.9 \\
\hline handlot & 0.38 & 0.31 & 4.99 & 8.3 & 12.5 & 13.3 & 71.7 \\
\hline sunlot & 0.37 & 0.31 & 4.99 & 6.7 & 7.5 & 12.5 & 96.7 \\
\hline cycle & 0.4 & 0.31 & 3.64 & 5.3 & 5.3 & 5.3 & 35.1 \\
\hline
\end{tabular}

are excluded the number of impostor scores using subset base is reduced. The reader must be aware of this fact while the performance measures of subset base and the other subsets are discussed.

The score distribution plots in Figs. 7.4 and 7.5 visually reveal that MC and PC achieve a better performance on the individual subsets as reported for the key point based method SIFT. The high overlap of genuine (coloured blue) and impostor (coloured red) score distribution is not only valid for the presented examples but also for all other considered subsets. The observations are in-line with the subsequent metric based quantification analysis of the results. Except for subset cycle a significant increase in the score distributions overlap for all acquisition condition changes can be clearly seen. The distribution plots of subset base (first row) and cycle show well-separated genuine and impostor comparison scores, only a small intersection area is present for all recognition schemes. This is proven by the performance measures EER, FMR ${ }_{100}, \mathrm{FMR}_{1000}$, zFMR and zFNMR as shown in Tables 7.1, 7.2, 7.3, 7.4 and 7.5, respectively. All the other subsets exhibit a much higher overlap between the score distributions, which again is proven by the other performance measures, in particular, described by an EER increase reporting a recognition performance decrease. For almost all other subsets and feature-extraction methods, the 
Table 7.3 Performance evaluation results for SIFT using the laser scanner data

\begin{tabular}{|c|c|c|c|c|c|c|c|}
\hline Name & avGen & avImp & EER & $\mathrm{FMR}_{100}$ & $\mathrm{FMR}_{1000}$ & zFMR & zFNMR \\
\hline \multicolumn{8}{|l|}{ Baseline } \\
\hline base & 0.11 & 0.02 & 5.28 & 19.8 & 34.5 & 34.5 & 43.6 \\
\hline \multicolumn{8}{|c|}{ External condition changes } \\
\hline humid & 0.04 & 0.01 & 20.62 & 52.5 & 65.8 & 74.2 & 78.5 \\
\hline light & 0.04 & 0.01 & 19.09 & 49.2 & 62.5 & 62.5 & 99.9 \\
\hline dark & 0.03 & 0.01 & 23.18 & 62.5 & 85.8 & 91.7 & 99.9 \\
\hline temp -5 & 0.04 & 0.01 & 28.20 & 73.3 & 83.3 & 86.7 & 99.9 \\
\hline temp +5 & 0.04 & 0.01 & 16.93 & 53.3 & 71.7 & 72.5 & 99.9 \\
\hline $\operatorname{skin} 10$ & 0.03 & 0.01 & 16.35 & 55.0 & 65.0 & 82.5 & 99.9 \\
\hline $\operatorname{skin} 25$ & 0.04 & 0.01 & 24.99 & 61.6 & 83.3 & 87.5 & 99.9 \\
\hline \multicolumn{8}{|c|}{ Subject-related condition changes } \\
\hline up5 & 0.03 & 0.01 & 44.54 & 48.5 & 63.2 & 67.1 & 99.9 \\
\hline up 10 & 0.04 & 0.01 & 43.01 & 46.5 & 59.4 & 63.8 & 99.9 \\
\hline tremb & 0.02 & 0.01 & 26.72 & 67.8 & 72.3 & 75.0 & 99.9 \\
\hline badpl & 0.02 & 0.01 & 29.23 & 93.5 & 99.9 & 99.9 & 99.9 \\
\hline bend & 0.04 & 0.01 & 18.94 & 51.7 & 68.1 & 68.9 & 99.9 \\
\hline tip & 0.06 & 0.01 & 8.47 & 19.6 & 78.6 & 88.8 & 99.9 \\
\hline trunk & 0.03 & 0.01 & 17.33 & 70.0 & 80.8 & 83.3 & 99.9 \\
\hline handlot & 0.04 & 0.01 & 20.75 & 56.6 & 67.5 & 73.3 & 99.9 \\
\hline sunlot & 0.02 & 0.01 & 28.08 & 78.3 & 87.5 & 89.1 & 99.9 \\
\hline cycle & 0.06 & 0.01 & 25.00 & 10.6 & 35.7 & 35.7 & 68.0 \\
\hline
\end{tabular}

genuine scores are shifted to the left as the number of low-valued genuine scores is higher compared to those of subset base or cycle. In these subsets, EER, FMR ${ }_{100}$, $F_{1000}$, zFMR and zFNMR values are higher as well. When comparing the laser and the LED scanner's score distribution plots (comparing Figs. 7.4 and 7.5) it can further be observed that there is hardly any difference in the detected overall trend of both scanners detectable. This suggests that the selected illumination module does not have an impact on the recognition process for the considered subsets and thus for the evaluated conditions.

The avGen and avImp scores do not show significant differences among the subsets. Furthermore, their values do not exhibit differences within each of the three recognition schemes, so it is not possible to distinguish between the different subsets. Thus, they do not provide any additional information regarding the impact of the various acquisition conditions. It is not possible to distinguish between the single subsets because the values belonging to one of the recognition schemes (MC, PC or SIFT) are nearly identical. This can be seen in column 2 and 3 of each of Tables 7.1, 7.2 and 7.3. Considering subsets humid till sunlot, the avGen values for MC, PC and SIFT are lower compared to the results of subset base and cycle. Further details upon the performance metrics for the data acquired by the laser scanner are displayed in Tables 7.1 (for MC), 7.2 (for PC) and 7.3 (for SIFT), respectively. 
Table 7.4 Performance evaluation results for MC using the LED scanner data

\begin{tabular}{|c|c|c|c|c|c|c|c|}
\hline Name & avGen & avImp & EER & $\mathrm{FMR}_{100}$ & $\mathrm{FMR}_{1000}$ & zFMR & zFNMR \\
\hline \multicolumn{8}{|l|}{ Baseline } \\
\hline base & 0.21 & 0.08 & 1.77 & 5 & 6.7 & 6.7 & 4.5 \\
\hline \multicolumn{8}{|c|}{ External condition changes } \\
\hline humid & 0.16 & 0.08 & 10.03 & 17.5 & 27.5 & 31.7 & 84.8 \\
\hline light & 0.12 & 0.07 & 25.58 & 49.2 & 60.8 & 70.8 & 100.0 \\
\hline dark & 0.16 & 0.08 & 7.50 & 15.8 & 25.0 & 25.0 & 96.1 \\
\hline temp -5 & 0.13 & 0.08 & 18.34 & 37.5 & 43.33 & 48.33 & 96.9 \\
\hline temp +5 & 0.16 & 0.08 & 11.80 & 21.7 & 26.7 & 32.5 & 99.9 \\
\hline $\operatorname{skin} 10$ & 0.16 & 0.08 & 7.69 & 9.4 & 17.0 & 18.8 & 96.5 \\
\hline $\operatorname{skin} 25$ & 0.16 & 0.08 & 7.50 & 15.0 & 30.0 & 36.7 & 98.3 \\
\hline \multicolumn{8}{|c|}{ Subject-related condition changes } \\
\hline up5 & 0.14 & 0.08 & 42.49 & 98.3 & 99.2 & 99.2 & 99.9 \\
\hline up 10 & 0.16 & 0.08 & 40.00 & 99.2 & 99.9 & 99.9 & 99.6 \\
\hline tremb & 0.15 & 0.08 & 10.91 & 29.4 & 41.2 & 42.9 & 94.8 \\
\hline badpl & 0.12 & 0.08 & 34.06 & 84.0 & 89.4 & 89.4 & 97.2 \\
\hline bend & 0.12 & 0.08 & 16.66 & 43.3 & 58.3 & 64.2 & 99.9 \\
\hline tip & 0.15 & 0.08 & 13.33 & 23.3 & 55.0 & 55.9 & 99.9 \\
\hline trunk & 0.11 & 0.08 & 6.66 & 16.7 & 21.7 & 23.3 & 58.9 \\
\hline handlot & 0.15 & 0.08 & 17.41 & 21.7 & 29.2 & 29.2 & 96.8 \\
\hline sunlot & 0.15 & 0.08 & 8.34 & 20.0 & 28.3 & 31.2 & 87.7 \\
\hline cycle & 0.17 & 0.08 & 2.05 & 1.5 & 3.0 & 3.5 & 4.0 \\
\hline
\end{tabular}

The performance measures for the LED setup are listed in Tables 7.4 and 7.5. The corresponding values of SIFT will not be displayed separately due to the low recognition performance of SIFT. The SIFT results are quite similar to the results of the laser lights scanner, which are presented in Table 7.3. To summarise the results it can be said that there is no difference regarding the overall trend between laser and LED concerning avGen and avImp. However, there are some differences regarding the considered performance metrics. First, the performance on the LED data is better compared to the laser subsets, especially for PC in the most cases. Second, the results exhibit bigger differences among the acquisition conditions. In particular, subset light, temp -5 and temp +5 are showing the highest number of FMR $\mathrm{F}_{100}$, $\mathrm{FMR}_{1000}$, zFMR and zFNMR values related to environmental condition changes, while the EER is worst for temp - 5. If non-environmental acquisition variations are taken into account, up5, up10 and badpl are the most influencing conditions. They exhibit much higher error measures as detected in the baseline results which indicates a much worse overall performance of these subsets.

Based on the EER, FMR $100, \mathrm{FMR}_{1000}$, zFMR and zFNMR results it can be summarised that the impact of varying acquisition conditions on the recognition performance seems to be influenced by (a) certain acquisition conditions and (b) the applied 
Table 7.5 Performance evaluation results for PC using the LED scanner data

\begin{tabular}{|c|c|c|c|c|c|c|c|}
\hline Name & avGen & avImp & EER & $\mathrm{FMR}_{100}$ & $\mathrm{FMR}_{1000}$ & zFMR & zFNMR \\
\hline \multicolumn{8}{|l|}{ Baseline } \\
\hline base & 0.42 & 0.31 & 0.84 & 0.8 & 1.7 & 1.7 & 1.3 \\
\hline \multicolumn{8}{|c|}{ External condition changes } \\
\hline humid & 0.4 & 0.31 & 2.49 & 2.5 & 2.5 & 3.3 & 91.6 \\
\hline light & 0.35 & 0.30 & 21.53 & 33.3 & 35.8 & 40.8 & 99.9 \\
\hline dark & 0.39 & 0.31 & 0.84 & 0.8 & 0.8 & 1.7 & 44.7 \\
\hline temp -5 & 0.37 & 0.30 & 7.50 & 10.8 & 11.7 & 14.2 & 79.7 \\
\hline temp +5 & 0.39 & 0.30 & 10.00 & 11.7 & 14.2 & 15.0 & 99.9 \\
\hline $\operatorname{skin} 10$ & 0.4 & 0.30 & 0.85 & 0.0 & 0.9 & 0.9 & 0.9 \\
\hline $\operatorname{skin} 25$ & 0.39 & 0.30 & 0.84 & 0.8 & 1.7 & 1.7 & 75.3 \\
\hline \multicolumn{8}{|c|}{ Subject-related condition changes } \\
\hline up5 & 0.38 & 0.3 & 49.15 & 98.3 & 99.9 & 99.9 & 97.2 \\
\hline up 10 & 0.39 & 0.31 & 43.33 & 99.9 & 99.9 & 99.9 & 98.8 \\
\hline tremb & 0.39 & 0.31 & 3.35 & 3.4 & 10.0 & 11.0 & 48.5 \\
\hline badpl & 0.34 & 0.3 & 27.64 & 51.1 & 57.4 & 61.7 & 99.8 \\
\hline bend & 0.38 & 0.3 & 9.15 & 11.7 & 13.3 & 17.5 & 99.6 \\
\hline tip & 0.35 & 0.31 & 6.66 & 7.5 & 13.3 & 15.0 & 93.7 \\
\hline trunk & 0.38 & 0.3 & 5.79 & 5.8 & 11.6 & 11.7 & 79.0 \\
\hline handlot & 0.38 & 0.31 & 9.18 & 12.5 & 12.5 & 12.5 & 98.5 \\
\hline sunlot & 0.38 & 0.3 & 3.33 & 4.2 & 4.2 & 5.0 & 74.5 \\
\hline cycle & 0.4 & 0.31 & 1.24 & 0.6 & 1.2 & 1.3 & 1.1 \\
\hline
\end{tabular}

recognition system. The recognition accuracy across all performed experiments is influenced by acquisition condition changes. Some display a high amount of performance degradation, while others hardly show any influence. Furthermore, it is not clear how the impact of the different acquisition variations will change if the number of available distorted FV images is increased. These first results-showing an impact on the recognition process using images acquired under varying conditions-may not necessarily be observed in a large database to the same extent. Nevertheless, we are quite sure that based on the first results, several of the considered acquisition conditions have a high impact on the FV recognition process. These especially include changes in ambient light and temperature as well as misplacement of the finger inside the scanner. In order to get a deeper insight in the particular influence of these conditions, we plan to extend the database in terms of subjects and acquired FV images to perform a more reliable analysis with respect to the influence of varying acquisition conditions on FV recognition systems in practical application scenarios. 


\subsection{Conclusion}

In this chapter, the influence of varying environmental and non-environmental acquisition conditions in FV recognition was evaluated. The main purpose was the quantification and selection of the most influencing factors in terms of recognition accuracy. It is known from other studies that many biometric recognition schemes are influenced by environmental and subject-related factors. However, this aspect has not been investigated comprehensively for FV recognition so far. We selected several promising environmental and non-environmental acquisition condition changes which are likely to influence the acquired vascular pattern images acquired under different acquisition conditions. The current investigation was designed to get some first results in order to identify the potentially most challenging condition changes. For this purpose, we established a first FV image database containing 18 different conditions in total. 60 images per condition have been acquired from 12 individual fingers. Although that only 12 fingers from 2 subjects have been acquired, the results are showing a clear trend. The evaluation, focusing on the quantification of false accepted and rejected comparisons, confirmed that several of the considered conditions have a severe influence on the recognition performance. The recognition performance is decreased for FV images acquired under varying conditions compared against images acquired under optimal conditions, which corresponds to practical applications of FV recognition systems including enrolment and authentication phase.

We identified several severe influencing conditions regarding the recognition performance. The highest influence is observable in subset up5 using LED lights and PC. Subsets badpl, light and temp - 5 were detected as most challenging conditions regardless of the type of the considered FV scanner devices. Thus, variations in environmental and non-environmental acquisition conditions can both result in severe FV recognition performance problems.

Based on these promising first results we plan to extend the acquired subset. It is mandatory to increase the number of subjects in order to gain a better insight into the issues with varying conditions during the application of FV recognition systems in daily life. We are confident that this will contribute to the development of FV recognition systems that are more robust against the influence of typical acquisition conditions present in practical deployments and lead to a more widespread use of FV biometrics in various everyday applications.

\section{References}

1. Fujifilm lens description. http://www.fujifilmusa.com/products/optical_devices/machinevision/2-3-15/hf9ha-1b/index.html. Accessed 16 July 2018

2. IDS camera description. https://de.ids-imaging.com/IDS/datasheet_pdf.php?sku=AB00184. Accessed 16 July 2018 
3. Baranoski Gladimir VG, Krishnaswamy Aravind (2004) An introduction to light interaction with human skin. Rev Inform Teor E Apl 11(1):33-62

4. Bashkatov AN, Genina EA, Kochubey VI, Tuchin VV (2005) Optical properties of human skin, subcutaneous and mucous tissues in the wavelength range from 400 to $2000 \mathrm{~nm}$. J Phys D Appl Phy 38(15):2543

5. Bickler PE, Feiner JR, Severinghaus JW (2005) Effects of skin pigmentation on pulse oximeter accuracy at low saturation. Anesthesiol J Am Soc Anesthesiol 102(4):715-719

6. Bowyer KW, Hollingsworth K, Flynn PJ (2008) Image understanding for iris biometrics: a survey. Comput Vis Image Underst 110(2):281-307

7. Cheong W-F, Prahl SA, Welch AJ (1990) A review of the optical properties of biological tissues. IEEE J Q Electron 26(12):2166-2185

8. Choi JH, Song W, Kim T, Lee S-R, Kim HC (2009) Finger vein extraction using gradient normalization and principal curvature. Proc SPIE 7251:9

9. Dhawan AP, D'Alessandro B, Fu X (2010) Optical imaging modalities for biomedical applications. IEEE Rev Biomed Eng 3:69-92

10. Hashimoto J (2006) Finger vein authentication technology and its future. In: 2006 symposium on VLSI circuits, 2006. Digest of Technical Papers. IEEE, pp 5-8

11. Kauba C, Uhl A (2015) Robustness evaluation of hand vein recognition systems. In: Proceedings of the international conference of the biometrics special interest group (BIOSIG'15). Darmstadt, Germany, pp 1-8

12. Kumar Ajay, Zhou Yingbo (2012) Human identification using finger images. IEEE Trans Image Proc 21(4):2228-2244

13. Kumar A, Hanmandlu M, Madasu VK, Lovell BC (2009) Biometric authentication based on infrared thermal hand vein patterns. In: 2009 digital image computing: techniques and applications. IEEE, pp 331-338

14. Lee EC, Lee HC, Park KR (2009) Finger vein recognition using minutia-based alignment and local binary pattern-based feature extraction. Int J Imaging Syst Technol 19(3):179-186

15. Lin Chih-Lung, Fan Kuo-Chin (2004) Biometric verification using thermal images of palmdorsa vein patterns. IEEE Trans Circuits Syst Video Technol 14(2):199-213

16. Lowe DG (1999) Object recognition from local scale-invariant features. In: Proceedings of the seventh IEEE international conference on computer vision (CVPR'99), vol 2. IEEE, pp 1150 $-1157$

17. Matsuda Yusuke, Miura Naoto, Nagasaka Akio, Kiyomizu Harumi, Miyatake Takafumi (2016) Finger-vein authentication based on deformation-tolerant feature-point matching. Mach Vis Appl 27(2):237-250

18. Miura Naoto, Nagasaka Akio, Miyatake Takafumi (2007) Extraction of finger-vein patterns using maximum curvature points in image profiles. IEICE Trans Inf Syst 90(8):1185-1194

19. Ortega-Garcia J, Fierrez-Aguilar J, Simon D, Gonzalez J, Faundez-Zanuy M, Espinosa V, Satue A, Hernaez I, Igarza J-J, Vivaracho C et al (2003) MCYT baseline corpus: a bimodal biometric database. IEEE Proc Vis Image Signal Proc 150(6):395-401

20. Prommegger B, Kauba C, Uhl A (2018) Longitudinal finger rotation-problems and effects in finger-vein recognition. In: Proceedings of the international conference of the biometrics special interest group (BIOSIG'18). Darmstadt, Germany, pp 1-11

21. Qin H, El-Yacoubi MA (2017) Deep representation-based feature extraction and recovering for finger-vein verification. IEEE Trans Inf Forensics Secur 12(8):1816-1829

22. Qin H, Li S, Kot AC, Qin L (2012) Quality assessment of finger-vein image. In: 2012 AsiaPacific Signal and information processing association annual summit and conference (APSIPA ASC). IEEE, pp 1-4

23. Ratha NK, Bolle RM (1998) Effect of controlled image acquisition on fingerprint matching. In: Proceedings of Fourteenth international conference on Pattern recognition, 1998, vol 2. IEEE, pp 1659-1661

24. Severinghaus JW, Koh SO (1990) Effect of anemia on pulse oximeter accuracy at low saturation. J Clin Monitor 6(2):85-88 
25. Simon-Zorita D, Ortega-Garcia J, Fierrez-Aguilar J, Gonzalez-Rodriguez J (2003) Image quality and position variability assessment in minutiae-based fingerprint verification. IEEE Proc Vis Image Signal Proc 150(6):402-408

26. Song W, Kim T, Kim HC, Choi JH, Kong H-J, Lee S-R (2011) A finger-vein verification system using mean curvature. Pattern Recognit Lett 32(11):1541-1547

27. Stewart RF, Estevao M, Adler A (2009) Fingerprint recognition performance in rugged outdoors and cold weather conditions. In: IEEE 3rd international conference on biometrics: theory, applications, and systems, 2009. BTAS'09. IEEE, pp 1-6

28. Xu J, Cui J, Xue D, Feng P (2011) Near infrared vein image acquisition system based on image quality assessment. In: 2011 international conference on electronics, communications and control (ICECC). IEEE, pp 922-925

29. Yang Jinfeng, Shi Yihua (2014) Towards finger-vein image restoration and enhancement for finger-vein recognition. Inf Sci 268:33-52

30. Lu Y, Yang G, Yin Y, Xi X (2017) Finger vein recognition with anatomy structure analysis. IEEE Trans Circuits Syst Video Technol

31. Zhang J, Yang J (2009) Finger-vein image enhancement based on combination of gray-level grouping and circular gabor filter. In: International conference on information engineering and computer science, 2009. ICIECS 2009. IEEE, pp 1-4

32. Zhao J, Tian H, Xu W, Li X (2009) A new approach to hand vein image enhancement. In: Second international conference on intelligent computation technology and automation, 2009. ICICTA'09, vol 1. IEEE, pp 499-501

33. Zheng H, Xu Q, Ye Y, Li W (2017) Effects of meteorological factors on finger vein recognition. In: IEEE international conference on identity, security and behavior analysis, ISBA 2017, New Delhi, India, February 22-24, 2017. pp 1-8

34. Zuiderveld K (1994) Contrast limited adaptive histogram equalization. In: Heckbert PS (ed) Graphics Gems IV. Morgan Kaufmann, pp 474-485

Open Access This chapter is licensed under the terms of the Creative Commons Attribution 4.0 International License (http://creativecommons.org/licenses/by/4.0/), which permits use, sharing, adaptation, distribution and reproduction in any medium or format, as long as you give appropriate credit to the original author(s) and the source, provide a link to the Creative Commons license and indicate if changes were made.

The images or other third party material in this chapter are included in the chapter's Creative Commons license, unless indicated otherwise in a credit line to the material. If material is not included in the chapter's Creative Commons license and your intended use is not permitted by statutory regulation or exceeds the permitted use, you will need to obtain permission directly from the copyright holder.

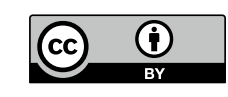

